\title{
Growing Cayley trees described by Fermi distribution
}

\author{
Ginestra Bianconi \\ Department of Physics, University of Notre Dame, Notre Dame,Indiana 46556,USA
}

\begin{abstract}
We introduce a model for growing Cayley trees with thermal noise. The evolution of these hierarchical networks reduces to the Eden model and the invasion percolation model in the limit $T \rightarrow 0, T \rightarrow \infty$ respectively. We show that the distribution of the bond strengths (energies) is described by the Fermi statistics. We discuss the relation of the present results with the scale-free networks described by Bose statistics.
\end{abstract}

\section{INTRODUCTION}

Recently it has been shown that Bose statistics [1] can be used to describe a scale-free network [2,3] with fitness of the nodes 堷. Since scale-free networks are continuously growing and develop a power-law connectivity distribution it is interesting to investigate their relation with self-organized processes [5 [7. In order to address this problem we present a model of invasion percolation with temperature defined on a Cayley tree, self-organized in the limit $T \rightarrow 0$. Our results show that the model can be solved analytically using the same technique used in the case of the scale-free networks described by Bose statistics.

The invasion percolation model [5] is the most famous and simple example of evolution with quenched disorder. It describes the displacement of a fluid in a porous medium. The porous medium is given by a random network constituted by bonds with different strengths $p$ chosen with uniform probability in the interval $(0,1)$. The classical asymptotic structure generated in this way is a fractal and the distribution of the strength values at the interface converges in time to a step function $\theta\left(p-p_{c}\right)$, where $p_{c}$ is the percolation threshold of the static percolation problem. In order to include the effect of fluctuations on the dynamics of invasion, present in a real stochastic cases, we include a temperature-like noise $T$ 88 11. A structure in which the invasion percolation dynamics can be defined is a Cayley-tree [12, 13], also known as the Bethe lattice. In this structure there are no loops, and the number of nodes in the bulk are of the same order of magnitude as the nodes at the interface. Therefore a Cayley tree is considered to be a good representation of a $d=\infty$ space and it is used in mean field calculations [14] and in the study of branching processes [15]. In this work we find that the distribution of bond strengths at the interface is no more a step function but it is described by the Fermi distribution with temperature $T$ where the bond strength plays the role of energy. By comparison with scale-free networks following the Bose statistics I show that both networks grow continuously in time: in the power-law network at each time a node is connected to the network by $m$ links while in the Cayley tree model at each time a node grows giving rise to $m$ new nodes. The dynamics of the two networks change in time award- ing the fitter nodes in the power-law network or choosing the less fit nodes to grow. At the same time in the powerlaw network the distribution of the energies of the chosen nodes converges to a Bose distribution while in a Cayley tree model the distribution of the energies at the interface converges to a Fermi distribution.

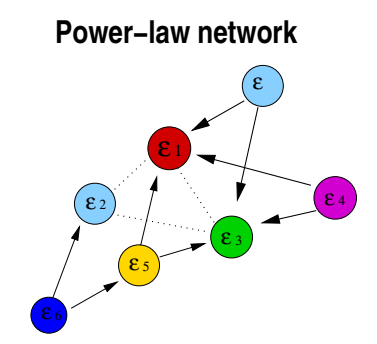

\section{Bose distribution}
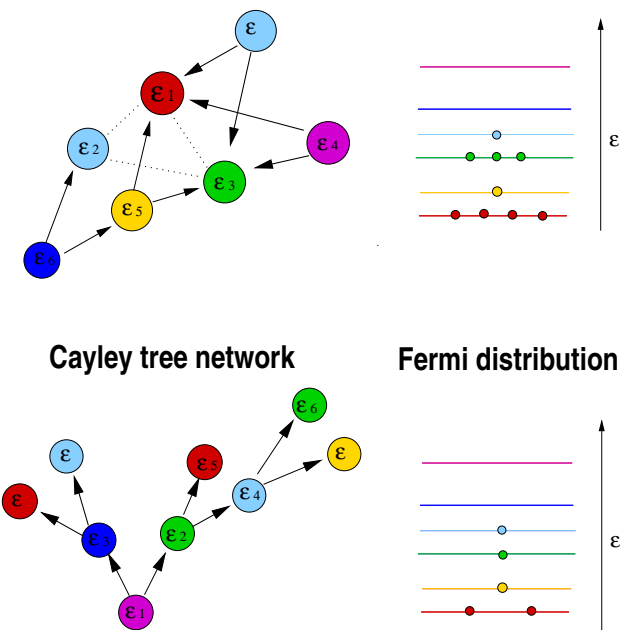

Fermi distribution

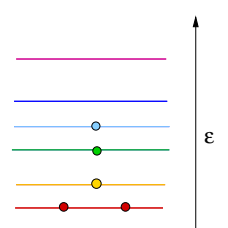

FIG. 1. Symmetric construction of a power-law network and the Cayley tree model considered in this paper.

\section{THE MODEL}

The Cayley tree (or Bethe lattice) is a loop-free network in which there are three classes of nodes: the root node, which is at the origin of the tree and has connectivity $m$, the nodes at the interface with connectivity one and the nodes in the bulk (below the interface) with connectivity $m+1$.

We start from the root of the tree (node $i=1$ ) and we link it to $m$ new nodes $i=2,3, \ldots, m+1$. We indicate each node with a subsequent number, $t_{i}$ indicating the time in which it is arrived in the interface.

At each timestep we choose one node to grow, giving rise to $m$ new nodes. Consequently, the interface of the tree grows linearly in time, and the growing node is chosen at each time from the growing number of active ones. In order to mimic the quenched noise of the medium we assign to each node of the tree an energy $\epsilon$ from a fixed random distribution $p(\epsilon)$. 
$\mathbf{t}=\mathbf{1}$
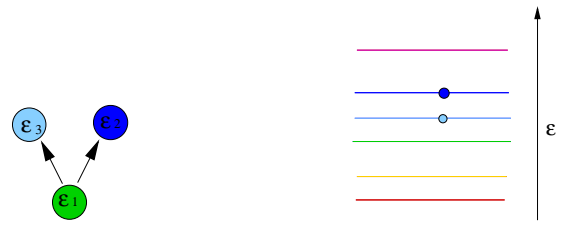

$\mathbf{t}=\mathbf{2}$
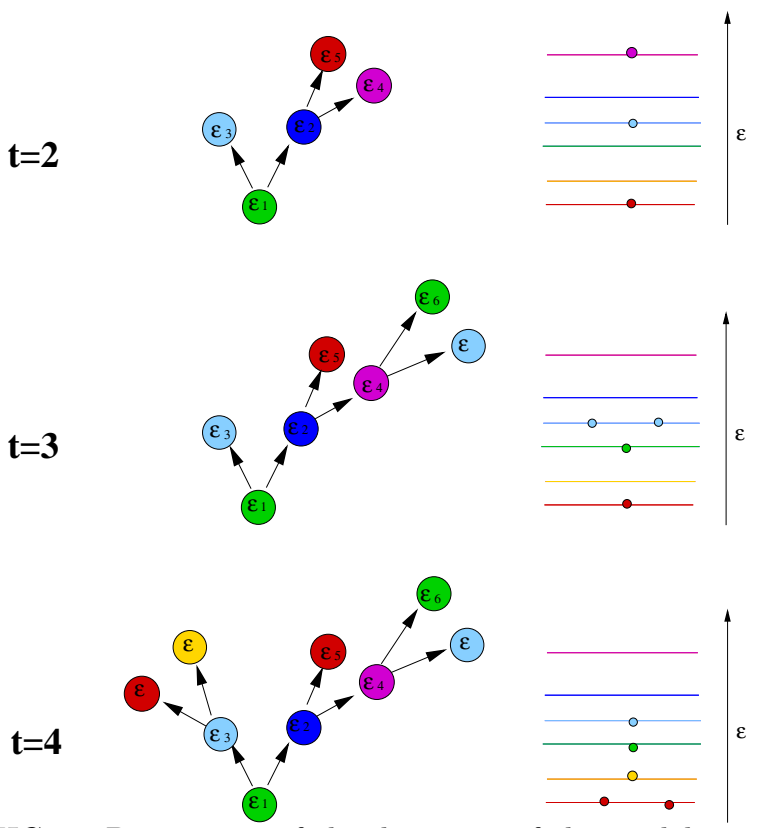

FIG. 2. Description of the dynamics of the model $m=2$. At time $t=1$ the root node $i=1$ with energy $\epsilon_{1}$ grows giving rise to $m$ new nodes $i=2,3$ with energies $\epsilon_{2}, \epsilon_{3}$. Node $i=1$ is in the bulk or below the interface (connectivity $m$ ) while the nodes $i=2,3$ have connectivity one and are at the interface. At time $t=2$ the node $i=2$ is chosen to grow, it leaves the interface giving rise to $m$ new nodes. $i=4,5$. At time $t=3,4$ nodes $i=4$ and $i=3$ are chosen to grow. In the right hand side of the figure we plot the density of states of the node in the interface. For example at time $t=4$ the nodes at the interface are $i=5,6,7,8,9$ and we can draw the density of states of the node at the interface by placing a particle in the energies levels $\epsilon_{5}=\epsilon_{8}, \epsilon_{9}<\epsilon_{6}<\epsilon_{7}$ as indicated in the picture.

We assume that higher energy nodes are more likely to grow than lower energy ones and that the probability $\Pi_{i}$ for the active node $i$ (with energy $\epsilon_{i}$ ) to grow at time $t$ is given by

$$
\Pi_{i}=\frac{e^{\beta \epsilon_{i}}}{\sum_{j \in \operatorname{Int}(t)} e^{\beta \epsilon_{j}}} .
$$

where the sum in the denominator is extended to all nodes $j$ that belong to the interface $\operatorname{Int}(t)$ at time $t$. The model depends on the parameter $\beta$. Tuning $\beta$ we change the nature of the model and the spatial aspect of the tree. In the $\beta \rightarrow 0$ limit, high and low energy nodes are equally probable to grow and the model reduces to the Eden model while in the $\beta \rightarrow \infty$ limit the dynamics becomes extremal such that only the nodes with the highest energy value are allowed to grow and the model reduces to invasion percolation [5] on a Cayley tree.

\section{EDEN MODEL ON A CAYLEY TREE}

Let us assume that every node has the same energy $\epsilon^{0}$, i. e. $p(\epsilon)=\delta\left(\epsilon-\epsilon^{0}\right)$. In this case all nodes at the interface are equally likely to grow and we call this model the Eden model on a Cayley tree. The probability that a node $i$ of the interface $\operatorname{Int}(t)$ grows at time $t$ is given by

$$
\Pi_{i}=\frac{1}{N_{\text {Int }}(t)},
$$

where $N_{\text {Int }}(t)$ is the total number of active nodes. Since at each timestep a node of the interface grows, becoming part of the bulk, and $m$ new active nodes are generated, after $t$ timesteps the model generates an interface of $N_{\text {Int }}(t)$ nodes, with

$$
N_{\text {Int }}(t)=(m-1) t+1
$$

We denote by $\rho\left(t, t_{i}\right)$ the probability that a node, born at time $t_{i}$ is still active at time $t$. Since every node grows with probability $\Pi_{i}$ Eq. 2 only if $i$ is a node of the interface, in mean field $\rho\left(t, t_{i}\right)$ follows

$$
\frac{\partial \rho\left(t, t_{i}\right)}{\partial t}=-\frac{\rho\left(t, t_{i}\right)}{N_{\text {Int }}(t)}
$$

Substituting (3) in (任) in the limit $t \rightarrow \infty$ we get the solution

$$
\rho\left(t, t_{i}\right)=\left(\frac{t_{i}}{t}\right)^{1 /(m-1)} .
$$

Consequently each node $i$ that arrives at the surface at time $t_{i}$, remains at the surface with a probability that decreases in time as a power-law. On the other side the same power-law describes also the distribution of the age $\tau$ of the nodes at the interface observed at time $t$. In fact, the probability $P(\tau)$ that a node born at time $\tau$ is still active at time $t$, is given by

$$
P(\tau)=\left(\frac{\tau}{t}\right)^{1 /(m-1)}
$$

Thus asymptotically in time the same power-law describes the time evolution of the nodes born at time $t_{i}$, $\left(\rho\left(t, t_{i}\right)\right)$ and the age distribution of the nodes in the interface, $(P(\tau))$. 


\section{Numerical simulations}

In order to verify the theoretical predictions, we have performed numerical simulations of the Eden model on a Cayley tree with $m=2,4,6$. In Fig. 3, we report the age distribution $P(\tau)$ of the nodes at the interface for Cayley trees with $m \times 10^{4}$ nodes and $m=2,4,6$. The data, averaged over 100 runs, follows the power-law predicted by (6). Numerical data are reported together with the theoretically predicted power-law (6).

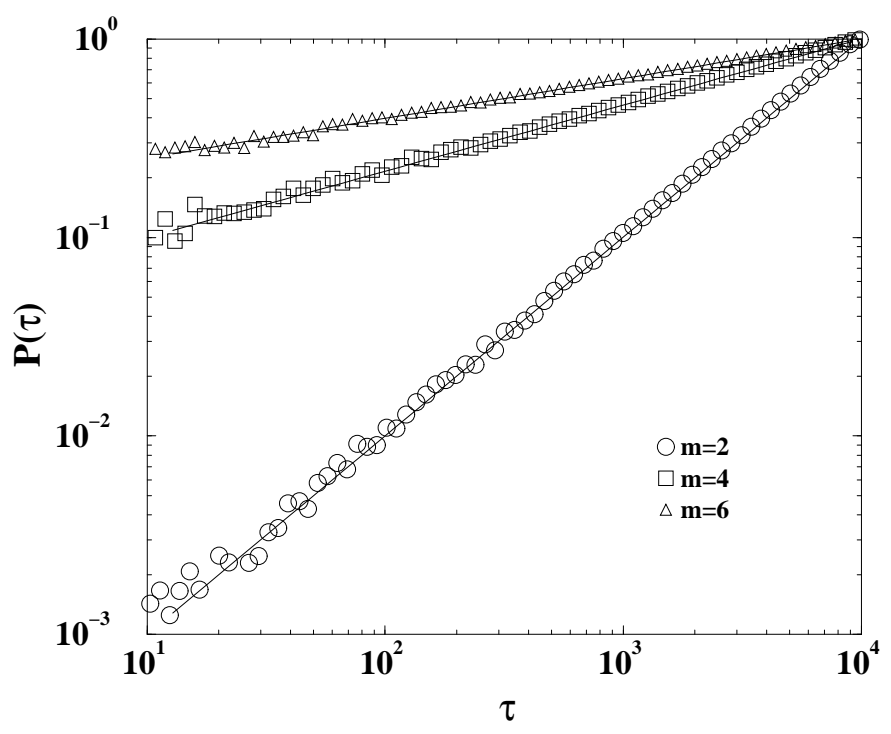

FIG. 3. Age distribution $P(\tau)$ of the nodes at the interface of a Cayley trees with connectivities $m=2,4,6$ and $10^{4}$ generations. Data have been averaged over 100 runs. The solid lines represent the power-laws predicted by (6) with exponent $1-1 / m$.

\section{CAYLEY TREE WITH ENERGIES}

At finite temperature, $\beta \neq 0$ it is necessary to take into account the fact that each node has a different energy that defines its dynamics. In this case the probability $\Pi_{i}$ for a node $i$ at the interface with energy $\epsilon_{i}$ to grow at time $t$ is given by (1)

$$
\Pi_{i}=\frac{e^{\beta \epsilon_{i}}}{\sum_{j \in \operatorname{Int}(t)} e^{\beta \epsilon_{j}}} .
$$

Since only nodes at the interface can grow, the probability that node $i$ would leave the interface at time $t$ is given by the product of $\rho_{i}\left(t \mid \epsilon_{i}, t_{i}\right)$ (the probability that the node is active at time $t$ ) and $\Pi_{i}$ (the probability that the node is chosen to grow in between the active nodes). Consequently, $\rho_{i}\left(t \mid \epsilon_{i}, t_{i}\right)$ decreases in time following

$$
\frac{\partial \rho\left(t \mid \epsilon_{t_{i}}, t_{i}\right)}{\partial t}=-\frac{e^{\beta \epsilon_{t_{i}}} \rho\left(t \mid \epsilon_{t_{i}}, t_{i}\right)}{\sum_{j \in \operatorname{Int}(t)} e^{\beta \epsilon_{j}}},
$$

were the sum in the denominator is extended to all the nodes $j$ that are part of the interface $\operatorname{Int}(t)$ at time $t$.

\section{Analytic solution}

To solve (8) we assume that in the thermodynamic limit the sum $Z^{S}$ in the denominator of the 1.h.s. of Eq. (8), given by

$$
Z^{S}=\sum_{j \in \operatorname{Int}(t)} e^{\beta \epsilon_{j}}
$$

self averages and converges to its mean value

$$
Z^{S} \rightarrow<Z^{S}>=\sum_{j=1, N} e^{\beta \epsilon_{j}} \rho_{j}\left(t \mid \epsilon_{j}, t_{j}\right) .
$$

Moreover, since $Z^{S}$ is an extensive quantity we can selfconsistently assume

$$
<Z^{S}>^{t \rightarrow \infty} z_{F} t\left(1+O\left(t^{-\alpha}\right)\right)
$$

Using (11), the dynamic equation (8) can be written as

$$
\frac{\partial \rho\left(t \mid \epsilon_{t_{i}}, t_{i}\right)}{\partial t}=-\frac{e^{\beta \epsilon_{i}}}{z_{F}} \frac{\rho\left(t \mid \epsilon_{i}, t_{i}\right)}{t} .
$$

Consequently we found that the time evolution of $\rho_{i}\left(t \mid \epsilon_{i}, t_{i}\right)$ follows a power-law,

$$
\rho_{i}\left(t \mid \epsilon_{i}, t_{i}\right)=\left(\frac{t_{i}}{t}\right)^{f\left(\epsilon_{i}\right)},
$$

but there is multiscaling in the system, i.e. the dynamic exponent depends on the energy $\epsilon_{i}$ of the node,

$$
f(\epsilon)=\frac{e^{\beta \epsilon}}{z_{F}} .
$$

After substituting $\rho_{i}\left(t \mid \epsilon_{i}, t_{i}\right)$ from Eq. (13) with $f(\epsilon)$ given by Eq. (14), into Eq. (11), and the sum with an integral, we get the self-consistent equation

$$
\begin{aligned}
<\sum_{j} e^{\beta \epsilon_{j}} \rho_{j}\left(t \mid \epsilon_{j}, t_{j}\right)>\stackrel{t \rightarrow \infty}{=} \int d \epsilon p(\epsilon) \int_{1}^{t} d t^{\prime} e^{\beta \epsilon}\left(\frac{t^{\prime}}{t}\right)^{f(\epsilon)} \\
=z_{F} t\left(1+O\left(t^{-\alpha}\right),\right.
\end{aligned}
$$

where

$$
\begin{gathered}
\alpha=\left(1+\min _{\epsilon} f(\epsilon)\right)>1, \\
z_{F}=m \int d \epsilon p(\epsilon) \frac{e^{\beta \epsilon}}{1+f(\epsilon)} .
\end{gathered}
$$

Finally, if we define $\mu_{F}$ as

$$
z_{F}=e^{\beta \mu_{F}}
$$


we obtain the self-consistent Eq. (17) can be interpreted as a definition of $\mu_{F}$ and it is formally equivalent to the definition of the chemical potential in an equilibrium Fermi gas

$$
1-\frac{1}{m}=\int d \epsilon p(\epsilon) \frac{1}{e^{\beta\left(\epsilon-\mu_{F}\right)}+1},
$$

suggesting that many properties of this model can be described by the Fermi statistics.

On the other hand the probability $P(\tau)$ that a node born at time $\tau$ is still active at time $t$, is given by a power-law

$$
P(\tau)=\int d \epsilon p(\epsilon)\left(\frac{\tau}{t}\right)^{e^{\beta\left(\epsilon-\mu_{F}\right)}} \sim\left(\frac{\tau}{t}\right)^{\delta}
$$

\section{Existence of the solution for the chemical potential}

Equation (19) always has a solution for the chemical potential $\mu_{F}$. In fact, since $p(\epsilon)$ is a normalized distribution function, the integral $I\left\{p(\epsilon), \mu_{F}\right\}$ on the r.h.s. of equation (19),

$$
I\left\{p(\epsilon), \mu_{F}\right\}=\int d \epsilon p(\epsilon) n_{F}(\epsilon)
$$

for a fixed $\mu_{F}$ is bounded by

$$
\frac{1}{e^{\beta\left(\epsilon_{\min }-\mu_{F}\right)}+1}<I\left\{p(\epsilon), \mu_{F}\right\}<\frac{1}{e^{\beta\left(\epsilon_{\max }-\mu_{F}\right)}+1}
$$

implying that for the chemical potential $\mu_{F}$ the solution of Eq.(19), $I\left\{p(\epsilon), \mu_{F}\right\}=1-1 / m$, satisfies

$$
\epsilon_{\min }+\frac{1}{\beta} \log (m-1)<\mu_{F}<\epsilon_{\max }+\frac{1}{\beta} \log (m-1) .
$$

This proves that for a real tree with $m>1$ the equation (19) always has a solution. Finally some attention should be given to the special limits $\beta \rightarrow 0$ and $\beta \rightarrow \infty$.

$\beta \rightarrow 0$ limit - In this case we recover the solution of the Eden model on the tree, $z_{F}=m-1$. Since the probability distribution $p(\epsilon)$ is normalizable and the occupation number

$$
n_{F}(\epsilon) \rightarrow \frac{1}{z_{F}^{-1}+1},
$$

equation (19) reduces to $z_{F} \rightarrow m-1$ and thus $\beta \mu_{F} \rightarrow$ $\ln (m-1)$ in such a way that $\mu_{F} \geq 0\left(z_{F}>1\right)$ if $m>2$.

$\beta \rightarrow \infty$ limit- In this limit the Fermi-Dirac distribution converges to the step function

$$
n_{F}(\epsilon) \rightarrow \theta\left(\epsilon-\mu_{F}\right)
$$

and the self-consistent equation (19) becomes

$$
1-\frac{1}{m}=\int_{\epsilon<\mu_{F}} p(\epsilon)
$$

\section{Mass conservation}

The self-consistent relation (19) can also be derived from mass conservation, i.e. from the knowledge that the total number of nodes at the interface is given by $N=(m-1) t$. Consequently,

$$
N=(m-1) t=\sum_{i \in[0, m t]} \rho\left(t \mid \epsilon_{i}, t_{i}\right)
$$

We can substitute the sum in the right hand side of Eq. (27) with the mean over the energies $\epsilon_{i}$ of the nodes $i$ of generation $t_{i}$. Moreover, in the thermodynamic limit we can approximate the sum over $i$ with an integral over $t_{i}$, the mass conservation relation becoming

$$
\begin{aligned}
(m-1) t & =m \int d \epsilon p(\epsilon) \int_{1}^{t} d \tau \rho(t \mid \epsilon, \tau) \\
& =m \int d \epsilon p(\epsilon) \int_{1}^{t} d \tau\left(\frac{\tau}{t}\right)^{e^{\beta\left(\epsilon-\mu_{F}\right)}} \\
& \simeq m t \int d \epsilon p(\epsilon) \frac{1}{e^{\beta\left(\epsilon-\mu_{F}\right)}+1}
\end{aligned}
$$

where in the last equation we have neglected terms of order $O\left(t^{-\alpha}\right)$. Thus both the mass conservation relation (27) and the self-consistent relation (19) allow us to define the chemical potential $\mu_{F}$, describing the evolution of the network as the chemical potential of an equilibrium Fermi gas with specific volume $v_{c}=1+1 /(m-1)$. However this last expression explains the meaning of that relation. In fact, the number $N_{\text {Int }}(\epsilon)$ of nodes with energy $\epsilon$ at the interface at time $t$ is given by

$$
N_{\text {Int }}(\epsilon)=\operatorname{mtn}_{F}(\epsilon) p(\epsilon)
$$

where $n_{F}(\epsilon)$ is given by the Fermi occupation number

$$
n_{F}(\epsilon)=\frac{1}{e^{\beta\left(\epsilon-\mu_{F}\right)}+1} .
$$

In other words, the distribution of the energy at the interface reaches a stationary limit given by (29) and defined by a Fermi distribution with chemical potential given by (19). In the mean time, the density of nodes with energy $\epsilon$ present in the bulk, $N_{\text {Bulk }}(\epsilon)$, reaches a stationary limit as well. In fact, since the nodes in the bulk are the ones of the network that are not at the interface, using (29), we have

$$
N_{B u l k}(\epsilon)=p(\epsilon)\left[1-n_{F}(\epsilon)\right]
$$




\section{Asymptotic dynamics}

The dynamical evolution of the network brings the system to the stationary state 16, 17] described by the distribution function (30), as it has been shown by the solution of the dynamical equation (8). Moreover the dynamics stabilizes this distribution. In fact, in the asymptotic limit, when the survivability follows (13) the probability that a node of energy $\epsilon$ will grow and leave the interface is given by

$$
\pi_{F}(\epsilon, t)=m \int d \epsilon^{\prime} p\left(\epsilon^{\prime}\right) \int_{1}^{t} d t^{\prime} \frac{\partial \rho\left(t \mid \epsilon^{\prime}, t^{\prime}\right)}{\partial t} \delta\left(\epsilon-\epsilon^{\prime}\right)
$$

which, using (12) can be extimated to be

$$
\begin{aligned}
\pi_{F}(\epsilon, t) & =\int d \epsilon^{\prime} m p\left(\epsilon^{\prime}\right) \int_{1}^{t} d t^{\prime} \frac{e^{\beta\left(\epsilon-\mu_{F}\right)} \rho\left(t \mid \epsilon^{\prime}, t^{\prime}\right)}{t} \delta\left(\epsilon-\epsilon^{\prime}\right) \\
& =m e^{\beta\left(\epsilon-\mu_{F}\right)} p(\epsilon) \frac{1}{t} \int_{1}^{t} d t^{\prime}\left(\frac{t^{\prime}}{t}\right)^{e^{\beta\left(\epsilon-\mu_{F}\right)}} \\
& \simeq m p(\epsilon)\left[1-n_{F}(\epsilon)\right] .
\end{aligned}
$$

Consequently, the probability that a node of energy $\epsilon$ leaves the interface, asymptotically in time, reaches a stationary limit independent of the particular evolution of the network, given by

$$
\pi_{F}(\epsilon, t) \rightarrow \pi_{F}^{*}(\epsilon)=p(\epsilon)\left[1-n_{F}(\epsilon)\right] .
$$

If we observe an evolving network and we have no knowledge of the age of the nodes, but only of their energies, the complete dynamics is determined by $\pi(\epsilon, t)$ describing which is the probability that a node with energy $\epsilon$ will leave the interface at time $t$. While the complete dynamics (8) is clearly dependent on time, $\pi(\epsilon, t)$, reaches the stationary limit $\pi_{F}^{*}(\epsilon)$, defining the invariant dynamics of the system.

The stability of the distribution $N_{b u l k}(\epsilon)$ of the energies in the bulk, is thus enforced by the dynamics. In fact we have found that asymptotically in time the probability that a node with energy $\epsilon$ is chosen to grow $\pi_{F}^{*}(\epsilon)$ is proportional to the number of nodes in the bulk $N_{b u l k}(\epsilon)$ given by Eq. (31).

\section{Numerical support}

Choosing the node energy from a uniform distribution $p(\epsilon)=1$ with $\epsilon \in[0,1]$, we have simulated the growth of a Cayley tree with $m=2$ and various values of $\beta$. In Fig. 4 we report the distribution of the energies of the active nodes for a network of size $N=2 \times 10^{4}$ nodes for $\beta=5,10,30$. The solid line in the figure represents the theoretical prediction described by (29) and (30) with a chemical potential given by $(19)$. In Fig. 5 the distribution of the age of the nodes at the interface is shown for $\beta=2,5,10,20$ and compared to the theoretical prediction Eq. (20), $P(\tau) \sim\left(\frac{\tau}{t}\right)^{\delta}$ with $\delta$ given by $e^{-\beta \mu_{F}}$ for the uniform distribution $p(\epsilon)=1, \epsilon \in[0,1]$.

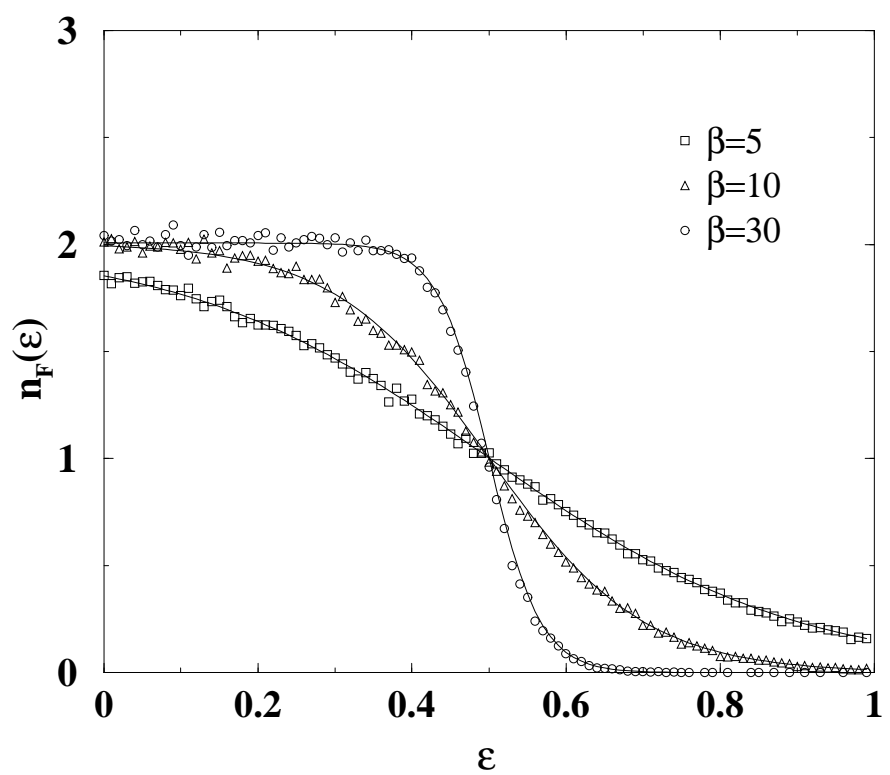

FIG. 4. The energy distribution of the nodes in the interface for $\beta=5,10,30$ in the case of a uniform energy distribution $p(\epsilon)=1$ for $\epsilon \in[0,1], m=2$ and predicted chemical potential $\mu_{F}=1 / 2$.The solid lines indicate the predicted Fermi distribution.

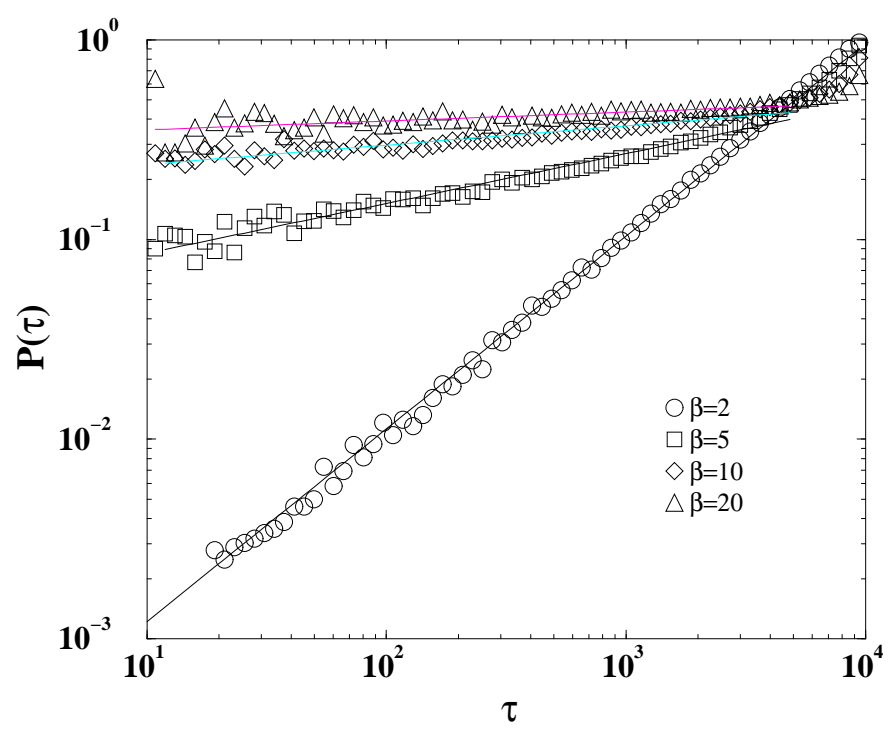

FIG. 5. Probability distribution of the age $\tau$ of the nodes at the interface in a Cayley tree with $m=2$ and time $10^{4}$ as a function of $\beta=2,5,10,20$.

\section{CONNECTION WITH OTHER PROBLEMS IN STATISTICAL MECHANICS}




\section{A. Invasion Percolation}

The static percolation problem on a Cayley tree is exactly solved and gives for the percolation threshold $p_{c}=1-1 / m$. The invasion percolation problem, represents the first example of a self-organized system and it has been solved exactly on a Cayley tree in [12] and numerically in 13 .

If we suppose that the energies of the tree model are uniformly distributed between zero and one, i.e. $p(\epsilon)=1$ for $\epsilon \in[0,1]$, in the limit $\beta=\infty$ the evolution of the interface of the Cayley tree network maps exactly into the front of invasion percolation on a Cayley tree. We can find the chemical potential $\mu_{F}$ by solving

$$
\begin{aligned}
1-1 / m & =\int d \epsilon n_{F}(\epsilon) \\
& =\int_{0}^{1} d \epsilon \frac{1}{e^{\beta\left(\epsilon-\mu_{F}\right)}+1} \\
& =\int_{0}^{\mu_{F}} d \epsilon=\mu_{F} .
\end{aligned}
$$

Consequently, asymptotically in time, the density of nodes with energy $\epsilon$ found at the interface follows the step function

$$
n_{F}(\epsilon)=\theta(\epsilon-(1-1 / m))
$$

predicting the correct threshold $p_{c}=1-1 / m$ for invasion percolation on a Cayley tree.

\section{B. Percolation}

The percolation transition can be investigated choosing a distribution function

$$
p(\epsilon)=p \delta(\epsilon-1)+(1-p) \delta(\epsilon)
$$

describing the fact that each node of the Cayley tree has energy $\epsilon=1$ with probability $p$ and energy $\epsilon=0$ with probability $1-p$. In the limit $T \rightarrow 0$ only the nodes with $\epsilon=1$ would grow. Consequently, assigning to the energy at the interface a meaning of cost function 18 we have that in the $T=0$ the mean energy of the node at the interface is minimized. When we consider the probability distribution (37), the self-consistent equation (19) reduces to a quadratic equation for the fugacity $z_{F}$,

$$
\left(1-\frac{1}{m}\right)=\frac{1-p}{z_{F}^{-1}+1}+\frac{p}{e^{\beta} z_{F}^{-1}+1} .
$$

that in the $\beta \rightarrow \infty$ limit has the solutions

$$
z_{F}^{-1}=\frac{-\Delta}{2\left(1-p_{c}\right)}\left(1 \pm \sqrt{1+4 e^{-\beta} \frac{p_{c}\left(1-p_{c}\right)}{\Delta^{2}}}\right)
$$

with $\Delta=\left(p-p_{c}\right)$ and $p_{c}=1 / m$. But only one solution is possible, because the fugacity is strictly positive. This implies that if $\Delta$ is positive $\left(p>p_{c}\right)$ we should take the negative sign in equation (39) while we have to use the positive sign for $p<p_{c}$.

Thus we have,

$$
z_{F} e^{-\beta} \simeq\left\{\begin{array}{ll}
e^{-\beta}\left(1-p_{c}\right) /|\Delta| & p<p_{c} \\
|\Delta| / p_{c} & p>p_{c}
\end{array} .\right.
$$

Consequently, using (37), the mean energy of the nodes at the interface is:

$$
\begin{aligned}
\epsilon_{\text {mean }} & =\frac{1}{m-1} \int d \epsilon \epsilon p(\epsilon) n_{F}(\epsilon) \\
& =\frac{p_{c}}{1-p_{c}} \frac{p}{e^{\beta} z_{F}^{-1}+1} .
\end{aligned}
$$

In the $T \rightarrow 0$ limit $\epsilon_{\text {mean }}$ displays a sharp transition at $p_{c}=1 / m$. Using the solution (40) in (41), we obtain

$$
\epsilon_{\text {mean }}= \begin{cases}e^{-\beta} p p_{c} /|\Delta| & p<p_{c} \\ \Delta /\left(1-p_{c}\right) & p>p_{c}\end{cases}
$$

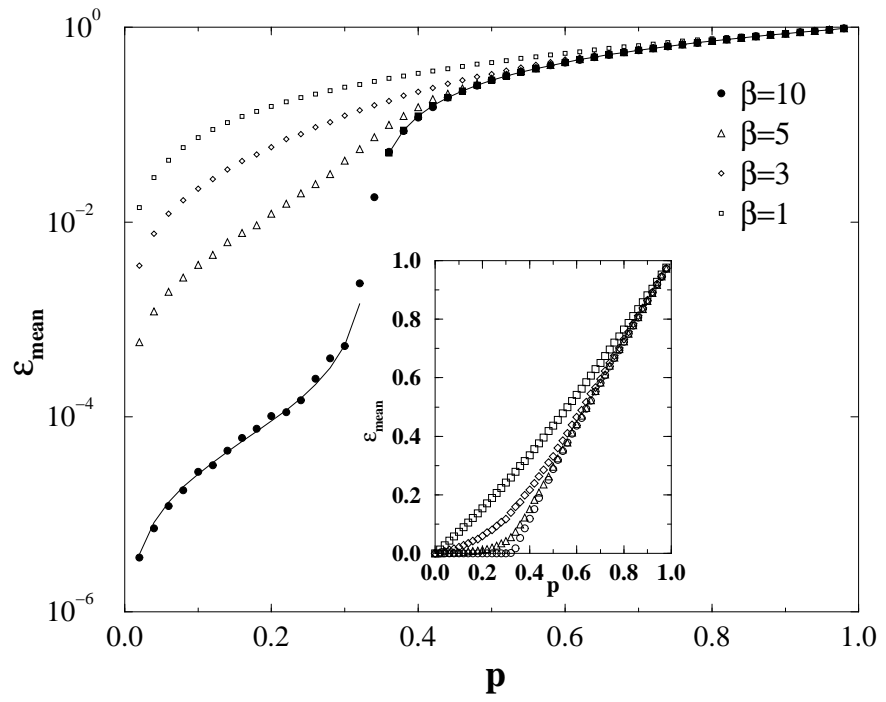

FIG. 6. Mean energy of the nodes at the interface as a function of $p$ and $\beta=10,5,3,1$. The solid line represent the theoretical prediction in the $\beta \rightarrow \infty$ limit.

In order to verify the predicted solution we have simulated the growth of a Cayley tree with $m=3$ branches. We have assigned to each node an energy equal to 0 or 1 with probability given by (37). The evolution of the network described by (11) proceeds preferentially on high energy nodes, in such a way that in the $\beta \rightarrow \infty$ the mean energy of the interface is minimized. In Fig. 6 we report the mean energy of the nodes at the interface as a function of $p$ for different temperatures. As the temperature goes to zero, the mean energy of the system has a sharp transition from zero to a non zero mean energy distribution. This shows that during the network evolution the nodes with high energy remain at the interface. 


\section{PARTITION IN TIME, ENERGY AND MEASURE}

\section{A. Partition in time}

If we coarse grain the time and divide the nodes following their age, we observe that the nodes with approximately the same age have a survivability $\rho\left(t, t_{i}\right)$ that grows in time as a power-law of $\ell_{i}=t / t_{i}$,

$$
<\rho\left(t, t_{i}\right)>_{\epsilon}=\int d \epsilon p(\epsilon) \rho\left(t, t_{i}, \epsilon\right) \simeq \ell_{i}^{-\tau_{F}}
$$

with $\tau_{F}$ asymptotically given by

$$
\tau_{F} \simeq e^{\beta \mu_{F}}
$$

This power-law describes the fact that the probability to be at the interface is lower for older nodes, but decreases much faster for nodes of energy $\epsilon>\mu_{F}$. The scale of the power-law depends on the ratio between the observation time and the time $t_{i}$ when the node $i$ joins the interface:

$$
\ell_{i}=\frac{t}{t_{i}}
$$

Because of this structure $\rho\left(t, t_{i}\right)$ is not a function separately of $t$ and $t_{i}$, and not even of their difference $t-t_{i}$, but only of their ratio $\ell_{i}$. Thus (43) describes both the probability that a node born at time $t_{i}$ is at the interface at time $t$ and the probability that a node at the interface at time $t$ is arrived in the network at time $t_{i}$.

Consequently the network at a given time will contain nodes of every age at different stages of their evolution. In other words the tree acts as if it was recording the evolution of the nodes, and the time partitions of the Cayley tree behaves as riscaled copies of the same network.

\section{B. Partition in energy}

A different case implies that the ages of the nodes are unknown but we have information about their energies. Thus it is natural to group together nodes with the same energy.

Since the total number of nodes with energy $\epsilon$ in the Cayley tree are $p(\epsilon) t$, the fraction of the nodes that remain at the interface is given by the integral of $\rho\left(t \mid t_{i}, \epsilon\right)$ over $t_{i}$, normalized to $p(\epsilon) t$, i.e.

$$
\frac{1}{p(\epsilon) t} \int d t_{i} p(\epsilon) \rho\left(t \mid t_{i}, \epsilon\right)=n_{F}(\epsilon) .
$$

As observed above this density is given by the Fermi occupation number $n_{F}(\epsilon)$, reached in the thermodynamic limit, and it gives the characteristic stable distribution of the Cayley tree evolution.

\section{The partition functions}

The emergence of the quantum statistics in the description of the geometrical structure of the interface of the Cayley tree suggests us the possibility to perform a more detailed investigation of its statistical properties.

\section{Static partition function}

In order to study the aspect of the Cayley tree at a given time, we can define the static partition function $Z^{S}(t)$ as

$$
Z^{S}(t)=\frac{\sum_{j \in \operatorname{Int}(t)} e^{\beta \epsilon_{j}}}{(m-1)}
$$

This describes the distribution of the energy values in the interface. As proven previously in (17), the static partition function, asymptotically in time, reaches a stationary limit and satisfies

$$
\lim _{t \rightarrow \infty} \frac{Z^{S}(t)}{t}=\frac{z_{F}}{(m-1)}
$$

Consequently, the static partition function defined in Eq. (47) is an extensive quantity related to the chemical potential of the network by

$$
\frac{1}{\beta} \lim _{t \rightarrow \infty} \frac{\log \left(Z^{S}(t)\right)}{t}=\mu_{F}-T \log (m-1) .
$$

\section{Dynamic partition function}

In order to describe the Cayley tree evolution we associate to it the sequence $\left\{\epsilon_{r}^{F}\right\}$ of the energy of the nodes selected to grow at time $t=r$. If we consider all the time dependent sequences associated to different realization of the networks for $t$ timesteps, we can associate with them a dynamical partition function $Z^{D}(t)$ given by

$$
Z^{D}(t)=\int \Pi_{r=1, t}\left\{d \epsilon_{r}\right\} e^{\beta \sum_{r=1}^{t} \epsilon_{r}} P\left(\epsilon_{1}, \epsilon_{2}, \ldots \epsilon_{t}\right)
$$

where the sum goes over all the different realizations of the network and $P\left(\epsilon_{1}, \epsilon_{2}, \epsilon_{m t}\right)$ is the probability of the sequence $\left\{\epsilon_{r}\right\}$. As we have shown previously (Eq. 34), in the thermodynamic limit the probability that an energy $\epsilon$ will be selected to grow reaches a stationary value given by the quantum occupation numbers. Consequently the partition function, asymptotically in time can be written as

$$
Z_{F}^{D}(t+1) \simeq Z_{F}^{D}(t) \int d \epsilon e^{\beta \epsilon} \pi_{F}^{*}(\epsilon)
$$


giving in the thermodynamic limit,

$$
Z^{D}(t) \simeq\left(z_{F}^{D}\right)^{t}
$$

were

$$
z_{F}^{D}=m \int d \epsilon p(\epsilon) e^{\beta \epsilon}\left[1-n_{F}(\epsilon)\right] .
$$

Thus for the induced dynamic in the energy space we can introduce a dynamical partition function describing the possible realization of the networks. The dynamical partition function, asymptotically in time is given by

$$
\lim _{t \rightarrow \infty} \frac{\log \left(Z_{F}^{D}(t)\right)}{t}=\log \left(z_{F}^{D}\right)
$$

The connection between the dynamical and the static fugacity is easily shown to be,

$$
(m-1) z_{F}^{S}+z_{F}^{D}=m<e^{\beta \epsilon}>_{p(\epsilon)}
$$

\section{Entropy}

One characteristic feature of this model is that the dynamics introduces a distinction between different nodes of the tree. In fact the nodes of the tree are distinguished between nodes in the interface and nodes in the bulk. The separation of this 'two phases' is forced by the dynamics itself and can be the reason for the statistical mechanics properties described by quantum statistics. In fact, if we look at the structure of the fermionic entropy $S$,

$$
\begin{gathered}
S=-\int d \epsilon p(\epsilon) n_{F}(\epsilon) \log \left(n_{F}(\epsilon)\right)+ \\
-\int d \epsilon p(\epsilon)\left[1-n_{F}(\epsilon)\right] \log \left(\left[1-n_{F}(\epsilon)\right]\right)
\end{gathered}
$$

we can observe that it can be interpreted as the sum of two Shannon entropies of the tree: the bulk and of the interface entropy,

$$
S_{F}=S_{i n t}+S_{b u l k} .
$$

\section{CONCLUSIONS}

In this work we have introduced a model for a growing Cayley tree with thermal noise characterized by

- Growth: At each time exactly $m$ nodes are added and one is eliminated at the interface, the number of nodes in which percolation can occur grows in linearly in time as $N=(m-1) t$ nodes;

- Time dependent dynamics: Each node can percolate only once and the probability for a node to be chosen as the percolating one is a decreasing function of time.
We have solved analytically the model studying its character in particular on the limit $\beta=0$ and then at finite temperature. The distribution of strength bonds follows a Fermi distribution and the dynamics asymptotically in time replicates and stabilizes this distribution. The bond strength plays the role of energies in the Fermi distribution. The distribution of ages of the node at the interface follows an effective power-law. We have then studied the cases in which the model reduces to the study of Invasion Percolation and percolation. We have found the percolation threshold and the mean energy of the growth. Finally we investigate the statistical properties of the solution and we define two partition functions describing the ensemble of growing Cayley trees. Finally this system is a symmetric construction of a power-law network following Bose distribution, as shown in Fig.1 and it opens the way to understand the self-organized nature of scale-free networks. 19.

\section{AKWNOLEDGEMENTS}

I would like to thank prof. A.-L. Barabási for support and help and L. Pietronero and A. Gabrielli for useful discussions.

[1] G. Bianconi, A.-L. Barabási, Phys. Rev. Lett. 86, 436 (2001).

[2] A.-L. Barabási and R. Albert, Science 286, 509 (1999).

[3] R. Albert and A.-L. Barabási, Rev. Mod. Phys. 74, 47 (2002).

[4] G. Bianconi, A.-L. Barabási, Europhy. Lett. 54 , 5632 (2001).

[5] D. Wilkinson and J. F. Willemsen, J. Phys. A 16, 3365 (1983).

[6] P. Bak, C. Tang and K. Wiesenfeld, Phys. Rev. Lett. 59,381 (1987).

[7] P. Bak and K. Sneppen, Phys. Rev. Lett. 71, 4083 (1993).

[8] M. Vergeles, Phys. Rev. Lett. 75, 1969 (1995)

[9] M. Vergeles, Phys. Rev. E 55, 6264 (1997).

[10] G. Caldarelli, A. Maritan and M. Vendruscolo, Europhys. Lett. 35, 481 (1996).

[11] A. Gabrielli, G. Caldarelli and L.Pietronero, Phys. Rev. E 62, 7638 (2000).

[12] B. Nickel and D. Wilkinson, Phys. Rev. Lett. 51, 71 (1983).

[13] N. Vandewalle and M. Ausloos Europhys. Lett. 37, 1 (1997).

[14] M. F. Thorpe, in Excitations in Disordered Systems, ed. M. F. Thorpe (Plenum press,1982). 
[15] T. E. Harris, The Theory of Branching Processes (Dover, New York, 1989).

[16] A. Erzan, L. Pietronero and A. Vespignani, Rev. Mod. Phys. 67545 (1995).

[17] R. Cafiero, A. Gabrielli, M. Marsili and L. Pietronero,
Phys. Rev. E 54, 1406 (1996)

[18] S. N. Majumdar and P. L. Krapivsky cond-mat/0006236 (2000).

[19] G. Bianconi, Int. Jour. Mod. Phys. B 14, 3356 (2000); ibidem, 15, 313 (2001). 